\title{
Tunable nano-replication to explore the omniphobic characteristics of springtail skin
}

\author{
René Hensel $^{1}$, Ralf Helbig ${ }^{1}$, Sebastian Aland ${ }^{2}$, Axel Voigt ${ }^{2}$, Christoph Neinhuis ${ }^{3,4}$ and Carsten Werner ${ }^{1,4}$ \\ Springtails (Collembola) are wingless arthropods adapted to cutaneous respiration in temporarily rain-flooded and microbially \\ contaminated habitats by a non-wetting and antiadhesive skin surface that is mechanically rather stable. Recapitulating the \\ robust and effectively repellent surface characteristics of springtail skin in engineered materials may offer exciting opportunities \\ for demanding applications, but it requires a detailed understanding of the underlying design principles. Towards this aim and \\ based on our recent analysis of the structural features of springtail skin, we developed a tunable polymer replication process to \\ dissect the contributions of different structural elements and surface chemistry to the omniphobic performance of the cuticle. \\ The Cassie-Wenzel transition at elevated pressures was explored by in situ plastron collapse experiments and by numerical FEM \\ simulations. The results obtained unravel the decisive role of nanoscopic cuticle structures for the protection of springtails \\ against wetting, and explain how the evolved nanotopography enables the production of omniphobic surfaces even from \\ intrinsically hydrophilic polymer materials.
}

NPG Asia Materials (2013) 5, e37; doi:10.1038/am.2012.66; published online 1 February 2013

Keywords: Collembola; cuticle; omniphobic; replication

\section{INTRODUCTION}

Springtails (Collembola), wingless arthropods are probably the most abundant hexapods on Earth ${ }^{1}$ and an integral component of the soil community $^{2}$ with more than 8000 species. ${ }^{3}$ Differing from insects, most springtail species respire through the skin. ${ }^{4,5}$ Consequently, their survival is affected by transpiration, that is critically dependent on environmental humidity. ${ }^{6,7}$ In turn, complete wetting of their skin blocks gas exchange and results in suffocation.

The consequent repellence of aqueous media evolved by the springtail skin has been known for more than half a century. ${ }^{6,8,9}$ However, the remarkable wetting resistance of the skin surfaces even against wetting by low-surface-tension liquids such as alkanes or ethyl alcohol and against bacterial adhesion were only recently quantitatively described. ${ }^{10}$ These findings reflect the adaptation to habitats where water is massively loaded with surface-active substances originating from decaying organic matter and from microorganisms.

A most striking feature of springtail skin is the hierarchically arranged and highly textured surface. ${ }^{9-11}$ Surface roughness has been extensively studied in general and shown to affect the macroscopic surface wetting properties significantly: In the Cassie state, liquids can be sustained at the top of the asperities and air (designated as plastron) is trapped inside the grooves of the rough surface underneath the liquid, resulting in a minimal solid-liquid contact area. ${ }^{12,13}$ In the Wenzel state, liquids can penetrate the grooves and wet the entire rough surface characterized by a maximal solid-liquid contact area. ${ }^{14}$ The metastable Cassie state can be transferred to the Wenzel state by means of supplied energy ${ }^{15,16}$ and geometric parameters of rough surfaces have been discussed to influence the energy barrier that must be overcome for complete wetting. ${ }^{17}$ Analysing the resulting wetting behaviour of rough surfaces is clearly more demanding if the surface roughness is hierarchically assembled with at least two sets of rough structures, ${ }^{18-20}$ as is common for many naturally occurring water-repellent surfaces. ${ }^{21-24}$ Therefore, the identification and mechanistic understanding of omniphobic and antiadhesive surface patterns evolved by living organisms still defines a challenge, where springtail skin represents a particularly interesting example.

Towards this aim, we have now adapted a nanoimprint lithography scheme as this recently developed methodology allows for the faithful replication of delicate morphologies with a few tens of nanometers resolution in polymeric materials of defined bulk and surface chemistry. ${ }^{25,26}$ When applying elastomeric moulds, the approach even permits the transfer of high aspect ratios, large overhangs ${ }^{27}$ and even closed loops. ${ }^{28}$ Successful replication of various different hierarchically assembled biological templates such as plant leaves, ${ }^{29}$ insect corneas $s^{30,31}$ or grasshopper wings ${ }^{32}$ was recently demonstrated.

Using this approach, we explored the role of distinct skin features in creating the unique omniphobic characteristics of springtail cuticle.

\footnotetext{
${ }^{1}$ Max Bergmann Center of Biomaterials, Leibniz Institute of Polymer Research Dresden, Dresden, Germany; ${ }^{2}$ Institute of Scientific Computation and Applied Mathematics, Technische Universität Dresden, Dresden, Germany; ${ }^{3}$ Institute of Botany, Technische Universität Dresden, Dresden, Germany and ${ }^{4} \mathrm{~B}$ CUBE Innovation Center for Molecular Bioengineering, Technische Universität Dresden, Dresden, Germany

Correspondence: Professor C Werner, Max Bergmann Center of Biomaterials, Leibniz Institute of Polymer Research Dresden, Dresden 01069, Germany.

E-mail: werner@ipfdd.de

Received 24 August 2012; revised 7 October 2012; accepted 2 November 2012
} 
A tunable nanoimprint replication process was developed to translate the skin structure of Tetrodontophora bielanensis (European giant springtail) into polymer replicas with defined variations in nanoscopic surface morphology and surface chemistry. We performed static contact angle measurements and determined the in situ wetting resistance at elevated pressures. In addition, the dynamics of the Cassie-Wenzel transition at the unravelled nanotopographies were investigated in detail by numerical FEM simulation and the results obtained were compared with experimental data.

With this approach, we were able to decipher the protection mechanism of springtail skin against wetting, and quantitatively determined the structural and chemical requirements for employing this design concept in the development of artificial structures.

\section{MATERIALS AND METHODS}

\section{Animals}

Tetrodontophora bielanensis springtails were collected in the wooded mountain of Saxony near Dresden, southeastern Germany. The animals were kept as laboratory colonies in large petridishes using soil, litter, decaying wood and moss from their original habitat as food source and substrate.

\section{Replication process}

Mould preparation. Individual animals were freshly prepared by freezing at $-20{ }^{\circ} \mathrm{C}$ for $30 \mathrm{~min}$ immediately before mould preparation was started. The animal was ventrally dipped into Fluorolink MD700 $\left(M W=1500 \mathrm{~g} \mathrm{~mol}^{-1}\right.$, Solvay Solexis, Bollate, Italy) precursor solution containing $0.5 \mathrm{wt} \%$ Irgacure 651 (CIBA, Basel, Switzerland) and mechanically fixed by exposing to UVirradiation 2 min by DELOLUX 04 (DELO, Windach, Germany) at each side. Next, Fomblin MD40 ( $M W=4000 \mathrm{~g} \mathrm{~mol}^{-1}$, Solvay Solexis) precursor solution containing $0.5 \mathrm{wt} \%$ Irgacure 651 was applied dorsally onto the animal skin and exposed to vacuum of $\sim 1 \mathrm{hPa}$ for $3 \mathrm{~h}$ to ensure infiltration of the entire skin by the precursor solution. Fomblin MD40 was crosslinked by exposing to UVirradiation for $5 \mathrm{~min}$ under nitrogen atmosphere. Subsequently, the cured elastomeric MD40 mould was gently peeled from the springtail skin.

Route A: Fabrication of faithful PEGda replicas. A PEGda $\left(\mathrm{M}_{n}=700 \mathrm{~g} \mathrm{~mol}^{-1}\right.$, Sigma Aldrich, Deisenhofen, Germany) precursor solution containing $0.5 \mathrm{wt} \%$ Irgacure 651 was cast onto the prepatterned MD40 mould and exposed to vacuum of $3 \times 10^{-3} \mathrm{hPa}$ for $12 \mathrm{~h}$. PEGda was crosslinked by exposing to UVirradiation for $5 \mathrm{~min}$ under nitrogen atmosphere. Subsequently, the PEGda polymer replica was gently peeled from the MD40 mould.

Route B: Fabrication of PEGda replicas without primary granules. A highviscous PDMS Sylgard 184 (Dow Corning, Wiesbaden, Germany) precursor solution was freshly mixed with the curing reagent in the ratio 10:1, cast onto the prepatterned MD40 mould and exposed to vacuum of $1 \mathrm{hPa}$ for $12 \mathrm{~h}$. The residuary PDMS crosslinking took place at room temperature over two more days. The elastomeric PDMS replica was gently peeled from the MD40 mould and used as template for the preparation of a further MD40 mould that, in turn, was used to make PEGda polymer replicas using the same procedure as described above in route $\mathrm{A}$.

\section{Surface treatment}

For Teflon-AF-coating, the PEGda polymer replicas were dipped ( $1 \mathrm{~min}$ ) into a solution of $0.6 \mathrm{wt} \%$ of amorphous fluoropolymer Teflon (Teflon AF, DuPont, Wilmington, DE, USA) diluted in a fully fluorinated solvent FC-77 (3M, Haven, Belgium). After withdrawing, the replicas were dried (10 min) on a hot plate at $60^{\circ} \mathrm{C}$.

\section{Contact angle measurements}

Static contact angle measurements were performed using the contact angle system OCA 30 (DataPhysics Instruments, Filderstadt, Germany). Droplets $(\sim 2 \mu \mathrm{l})$ of Milli-Q filtered water (Merck Millipore, Billerica, MA, USA) and hexadecane (Sigma-Aldrich) were dorsally applied at the skin of T. bielanensis and its polymer replicas. Dynamic contact angle measurements of the
Collembola skin and its polymer replicas were impossible due to the restricted sample area. Smooth reference polymer films were used for the determination of the intrinsic contact angles using dynamic contact angle measurements. The static contact angle was estimated as $\Theta_{\text {stat }}=\arccos \left(\frac{\cos \Theta_{\text {adv }}+\cos \Theta_{\text {rec }}}{2}\right)$ where $\Theta_{\text {stat }}$ is the static contact angle, $\Theta_{\text {adv }}$ is the advancing contact angle, and is $\Theta_{\text {rec }}$ the receding contact angle.

\section{In situ plastron collapse tests}

Plastron collapse experiments were performed using a self-made setup that consisted of an optical microscope, a water pump, a processing unit and a water-flooded chamber where the samples were previously placed ( $c f$. Figure 3a). The liquid reservoir was linearly compressed $50 \mathrm{hPas}^{-1}$ by increasing hydrostatic pressure and images were simultaneously recorded using an optical microscope unit. To minimize the influence of the gas solubility in water, the experiments were carried out at a water-air ratio of 10:1.

\section{SEM and TEM imaging}

Scanning electron microscopy studies were performed using a Gemini DSM 982 (Carl Zeiss SMT, Oberkochen, Germany). The animals were prepared by freezing and subsequent air-drying without any fixation. All samples (animals, moulds and replicas) were coated with $3 \mathrm{~nm}$ platinum (BAL-TEC SCD 500, BalTec, Pfäffikon, Switzerland) to eliminate surface charging effects. Transmission electron microscopy studies were carried out using an EM 912 Omega (Carl Zeiss SMT). The samples were fixed, stained and subsequently sliced into ultrathin sections as described in Helbig, R. et al. ${ }^{10}$

\section{RESULTS}

The hierarchical structure of the skin of T. bielanensis is depicted in Figure 1. At the nanoscopic level, primary granules are joined by interconnecting ridges covering the entire body of the springtail in a rhombic comb-like lattice. They form nanoscopic cavities with a mean diameter of about $300 \mathrm{~nm}$. The primary granules protrude above the ridges so that, in sectional view, the nanocavities exhibit overhangs. The skin at the bottom of these cavities has to be highly permeable to enable skin respiration. ${ }^{9}$ At the microscopic scale, T. bielanensis possess papillose secondary granules and a limited number of thin bristles as a tertiary structure. The secondary granules are completely decorated by primary granules excluding the smooth domes at the top of these papillae.

In order to resolve the impact of the individual structure elements on the characteristics of the springtail skin, we developed an adaptive replication process that is depicted schematically in Figure 2a (for more SEM images of each replication step, see the Supplementary Section 1 and Supplementary Figure S1). Starting from the biological template, T. bielanensis, the whole skin features were firstly captured by an elastomeric perfluoropolyether dimethacrylate (PFPEdma) mould. The mould material has a high fluorine content $\sim 58 \mathrm{wt} \%$ that inhibited chemical crossreactions with the biological template due to its chemical inertness. ${ }^{31}$ The PFPEdma mould as the negative replica of the natural skin was then the starting point for the fabrication of polymer skin replicas by two different routes. In route A, poly(ethylene glycol) diacrylate (PEGda) was used as a liquid prepolymer. After crosslinking the PEGda and subsequent demoulding, the polymer replicas possessed the entire superficial granular surface structure of T. bielanensis but did not contain any bristles (see Supplementary Figure S2). In route B, polydimethylsiloxane (PDMS) was used as the liquid prepolymer, with a significantly higher viscosity. The penetration of the more viscous prepolymer into the smallest pores of the mould was nearly inhibited (for more details, see Supplementary Section 2). Thus, the replicas of route B did not contain the nanoscopic primary granules and nanocavities. Furthermore, the generated PDMS replicas were used as templates for 


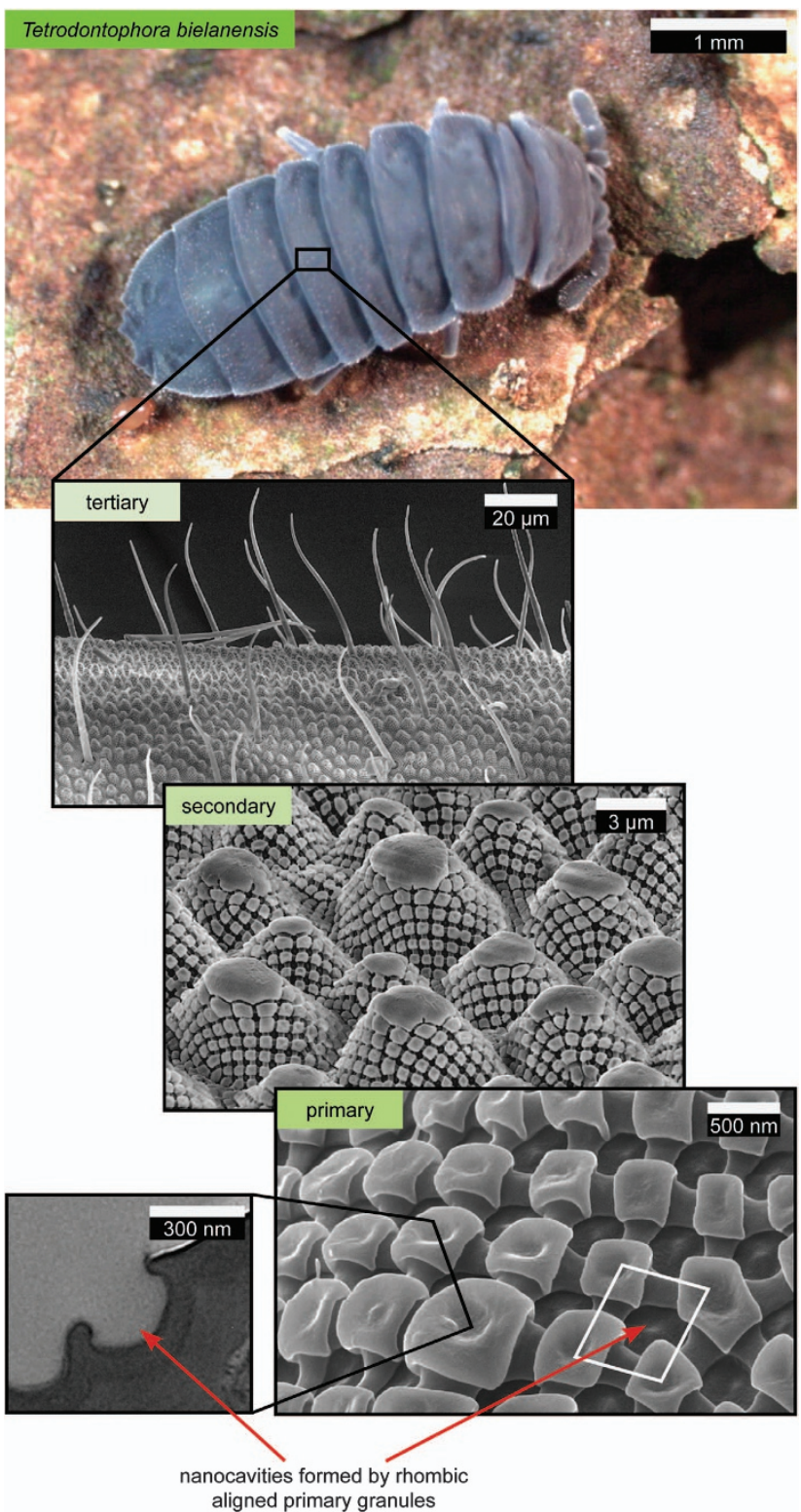

Figure 1 Skin pattern of $T$. bielanensis (European giant springtail). The inserts represents SEM images of the several skin features of the hierarchically structured skin. The smallest elements are primary granules that are joined by interconnecting ridges and spatially arranged in a rhombic comb-like lattice. Transmission electron micrograph of a section through the skin shows a nanocavity (diameter $\sim 300 \mathrm{~nm}$ ) that exhibits overhangs formed by adjacent primary granules. $T$. bielanensis possess papillose secondary granules and a limited number of thin bristles as secondary and tertiary structures, respectively. The secondary granules are completely decorated by primary granules excluding the smooth domes at the top of these papillae.

making replicas from PEGda polymer, the same material as used in route $\mathrm{A}$, which, of course, did also not contain primary granules. The crosslinked PEGda polymer is intrinsically hydrophilic, that is, wettable by water. A second set of polymer replicas was produced according to route $\mathrm{A}$ and route $\mathrm{B}$ as described above but additionally coated by amorphous Teflon (Teflon AF). This fluoropolymer is intrinsically hydrophobic due to its low surface energy of about $\sim 13.6 \times 10^{-3} \mathrm{~J} \mathrm{~m}^{-2} .33 \mathrm{In}$ sum, we produced polymer skin replicas of similar chemical bulk composition, but with distinctive surface
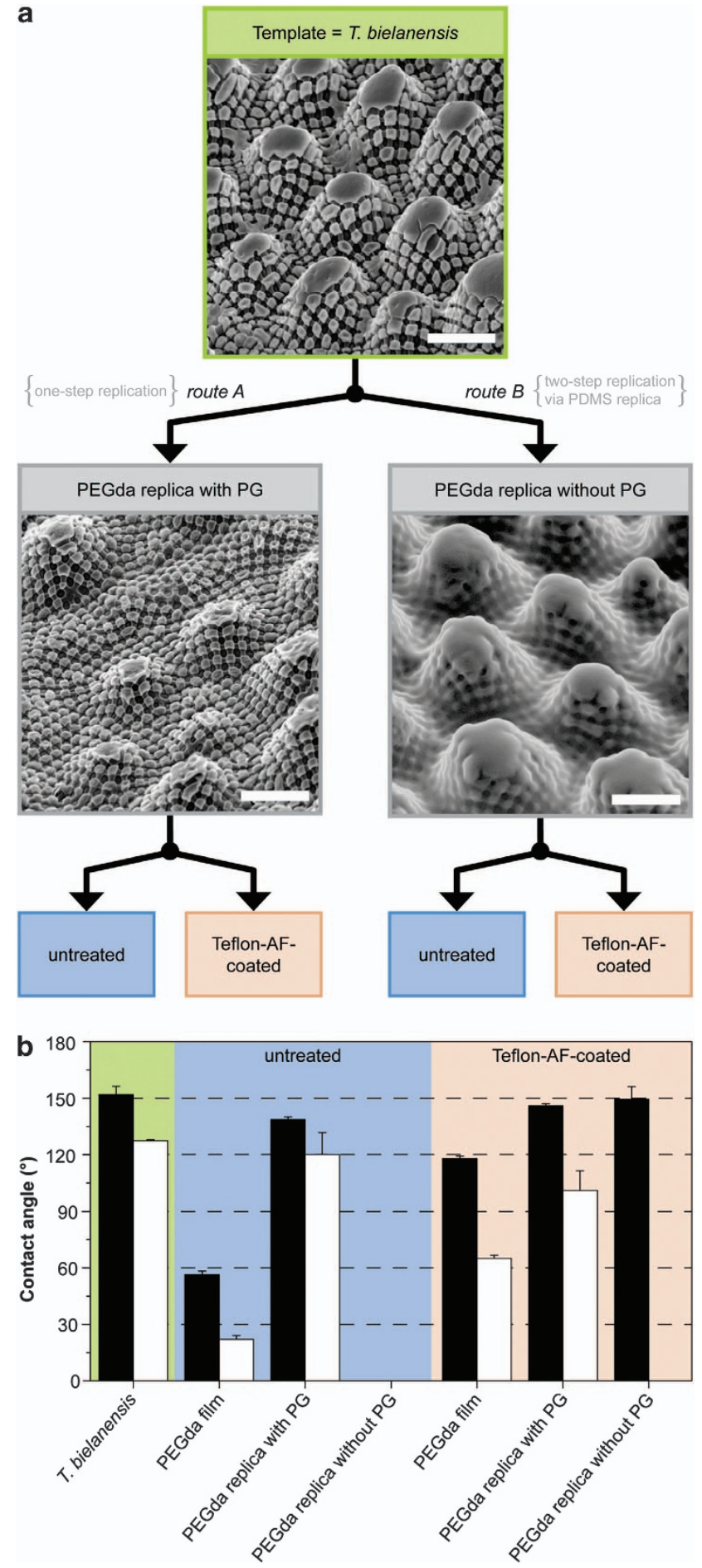

Figure 2 Replication process flow and the contact angle measurements. (a) Schematic illustration of the sample preparation via replication of the natural skin of $T$. bielanensis. Two routes are feasible albeit distinctive regarding the nanoscopic morphology; route $A$ generates faithful polymer skin replicas with primary granules, whereas route B leads to polymer replicas without primary granules. The material of the final replicas (in both routes) is poly(ethylene glycol) diacrylate (PEGda), a hydro and lyophilic polymer. In a further step, the surface chemistry is varied between untreated and Teflon-AF-coated polymer replicas. Scale bars: $3 \mu \mathrm{m}$. (b) Determined static contact angles using droplets of water (black bars) as a polar liquid with high surface tension and hexadecane (white bars) as a non-polar liquid with low surface tension. 
morphologies regarding the presence of the primary granules and distinctive surface chemistries.

The wetting of the generated polymer skin replicas was studied with contact angle goniometry using the skin of T. bielanensis, and smooth polymer films of the respective replica materials as references. Water was applied as a polar liquid with high surface tension $\left(\gamma=72.3 \times 10^{-3} \mathrm{~N} \mathrm{~m}^{-1}\right)$ and hexadecane as a non-polar liquid with low surface tension $\left(\gamma=27.5 \times 10^{-3} \mathrm{Nm}^{-1}\right)$. The results of the contact angle measurements are depicted in Figure $2 \mathrm{~b}$. The smooth films were used to determine the intrinsic contact angles, $\Theta_{Y}$, of the polymer surfaces without any influences of the surface roughness reflecting the moderately hydrophilic $\left(\Theta_{Y, \text { water }} \cong 56^{\circ}\right)$ and lyophilic $\left(\Theta_{Y, \text { hexadecane }} \cong 22^{\circ}\right)$ behaviour of PEGda polymer. Teflon-AF-coated polymer surfaces were intrinsically hydrophobic $\left(\Theta_{Y, \text { water }} \cong 118^{\circ}\right)$ and moderately lyophilic $\left(\Theta_{Y \text {,hexadecane }} \cong 65^{\circ}\right)$. The contact angle data of the replica samples showed a clear correlation with the distinctive surface morphologies: Polymer replica surfaces containing primary granules produced contact angles considerably $>90^{\circ}$ with values up to $150^{\circ}$ for both test liquids, reflecting an omniphobic wetting performance irrespective of the polymer surface chemistry. In contrast, PEGda polymer replicas without replication of the nanoscopic surface morphology of the springtail skin were completely soaked by both test liquids resulting in macroscopic contact angles of about $0^{\circ}$. Teflon-AF-coating of the latter replicas afforded water repellence but were soaked by hexadecane.

Thus, T. bielanensis and its faithful polymer replicas exhibited an omniphobic wetting performance irrespective of their surface chemistry based on the Cassie state of the liquid exposed to the surface, that is, a minimized liquid-solid contact area and a plastron underneath the liquid phase. The presence of such a plastron is
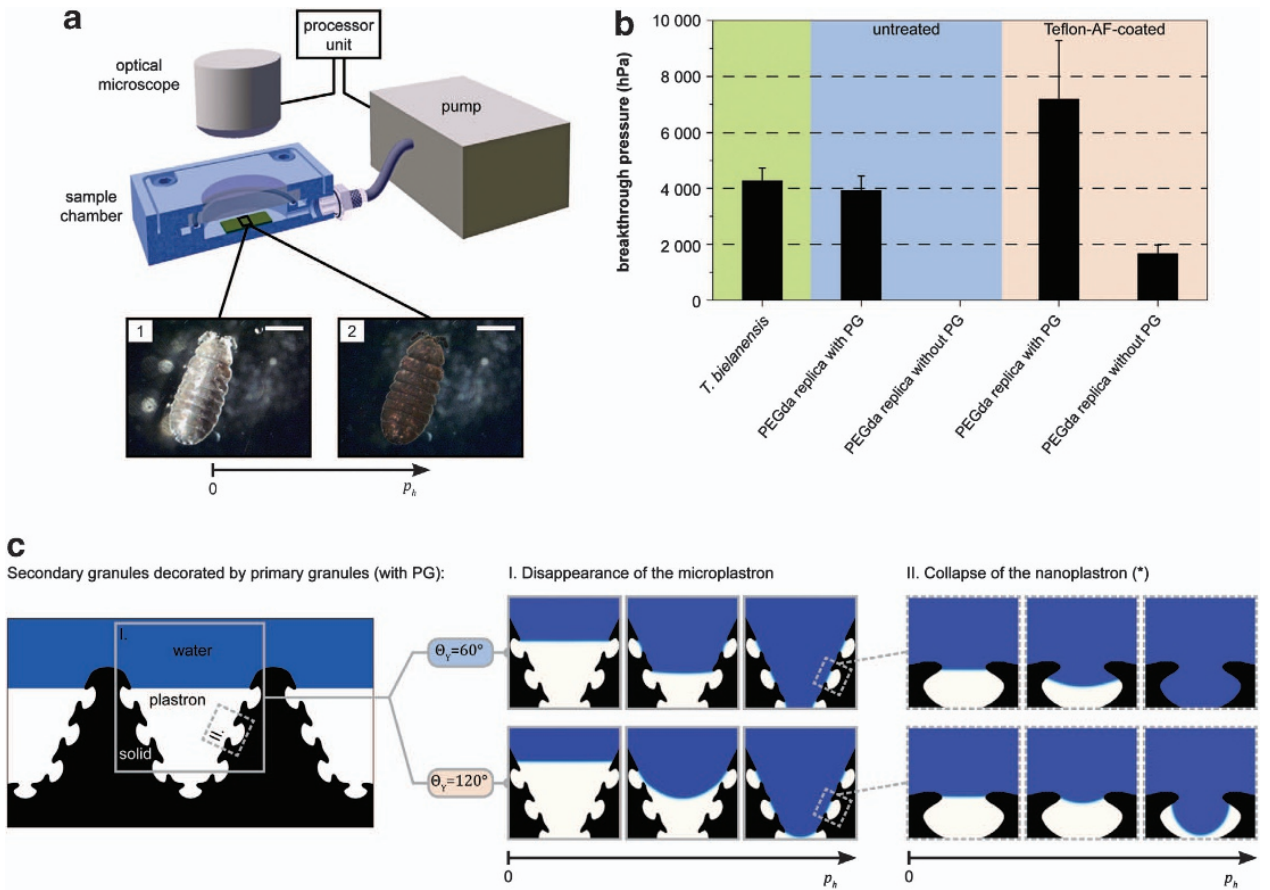

Secondary granules without primary granules (without $P G$ ):
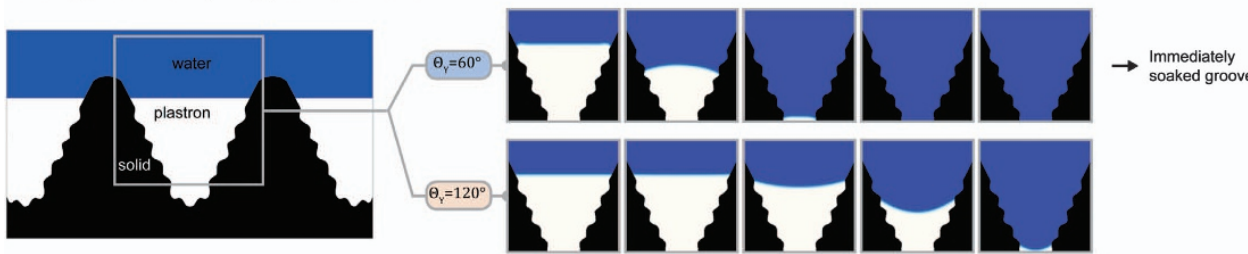

Figure 3 Pressure resistance and dynamics of the Cassie-Wenzel transition. (a) Schematic illustration of the experimental setup that consists of an optical microscope, a water pump, a processing unit and a water-flooded chamber (cross-sectional view) where the samples (green) were previously placed. Insert (1) shows the plastron (air cushion) that was formed around $T$. bielanensis by a visible shiny appearance. The disappearance of the shine was observed in situ using an optical microscope while at the same time the hydrostatic pressure inside the aqueous phase was linearly increased. Insert (2) shows the animal after final plastron collapse. Scale bars: $1 \mathrm{~mm}$. (b) Determined breakthrough pressures that provide the robustness of the Cassie state a priori of the set of polymer skin replicas in reference to $T$. bielanensis. (c) Numerical FEM-simulations to explore the dynamics of Cassie-Wenzel transition. On the left hand side, a sectional view through secondary granules that are either decorated by primary granules or not and the initial position of the fluid interface is schematically represented. On the right hand side, numerical results are presented by snap shots from left to right that display the advancing liquid front (blue) displacing the plastron (white) inside certain complex-shaped solid phase fields (black). Both hydrophilic $\left(\Theta_{Y}=60^{\circ}\right)$ and hydrophobic $\left(\Theta_{Y}=120^{\circ}\right)$ surfaces were considered similar to the experimental setup in our simulations. The hydrostatic pressure difference $p_{\text {top }}-p_{b o t t o m}=p_{h}$ between the top and bottom boundary was imposed as boundary conditions, which pushes the fluids downwards for $p_{h}>0$. $\left(^{*}\right)$ The numerical results that are denoted by collapse of the nanoplastron are independent simulations regardless of the dissapearance of the microplastron and elaborate on the final collapse of the remaining plastrons inside the nanocavities. 
obvious from its shiny appearance due to the total internal reflection of light at the liquid-air interface (see insert 1 in Figure 3a). With elevated pressures, the plastron structures gradually disappear, defining a measure for the Cassie-Wenzel transition of the respective surface structure. Plastron collapse tests were performed in situ to explore the robustness of the observed Cassie state of liquid in contact with $T$. bielanensis and the derived polymer replicas. The experimental setup of the applied pressure chamber is depicted schematically in Figure 3a. Upon immersion of the samples, the plastron was immediately formed and its shine gradually disappeared with increasing hydrostatic pressure (see Supplementary Movie S1 and S2). The pressure at which the plastron finally collapsed was defined as breakthrough pressure and the values are depicted in Figure $3 \mathrm{~b}$. The plastron formed around T. bielanensis collapsed at pressures of about $4200 \mathrm{hPa}$. Nearly the same value was determined for the untreated, faithful PEGda polymer replicas with pressures of about $3900 \mathrm{hPa}$. The breakthrough pressure values for the Teflon-AF-coated polymer replicas containing nanoscopic granules even exceeded those observed for the animals and were determined to $7200 \mathrm{hPa}$. In contrast, the untreated PEGda polymer skin replicas without primary granules were readily soaked after dipping into the aqueous phase. Teflon-AF-coating of these structures enabled the formation of a plastron but resulted in a significantly lower hydrostatic resistance of about $1800 \mathrm{hPa}$. Thus, the durability of the Cassie state clearly depends on both the nanoscopic morphology and the surface chemistry. The primary granules evolved by the springtail skin were found to afford the Cassie state even for intrinsically hydrophilic surfaces and the resistance against wetting at elevated pressures was enhanced by a factor of four when these replica surfaces were coated with Teflon AF.

As obvious from the experimental data obtained, the resistance of the springtail skin against wetting is strongly influenced by its particular nanotopography, which was now successfully replicated in faithful polymer structures. In order to gain further insights into the design principle of the skin resulting in the protective effect, the dynamics of the experimentally observed Cassie-Wenzel transition were analysed further by numerical FEM-simulations. With this approach, we specifically considered the enforced wetting of the nanoscopic patterned groove between two adjacent secondary granules. The two-dimensional simulations are based on a diffuse interface approach ${ }^{34}$ (for further details, see the Supplementary Section 3). As boundary conditions, we imposed a hydrostatic pressure difference $p_{\text {top }}-p_{\text {bottom }}=p_{h}$ between the top and bottom boundary, which pushed the fluids downwards for $p_{h}>0$. Furthermore, the considered solid phase fields were kept open at the bottom to focus on the impact of the geometric parameters of the solid surface by neglecting the effects of trapped air inside a closed cavity. Similar to the experimental setup, both hydrophilic $\left(\Theta_{Y}=60^{\circ}\right)$ and hydrophobic $\left(\Theta_{Y}=120^{\circ}\right)$ surfaces were considered in our simulations. Figure $3 \mathrm{c}$ illustrates the initial situation and the Cassie-Wenzel dynamics with a few transition states. Neglecting gravity and assuming that the length scale of the surface roughness remains below the capillary length of the applied aqueous phase, the fluid interface between the water and the plastron can be considered as approximately planar in the equilibrium state $\left(p_{\text {top }}=p_{\text {bottom }}\right)$. Starting the experiment by continuously increasing the hydrostatic pressure, the three-phase contact line was found to propagate downwards until the edges of the first overhangs were reached. At these edges, the three-phase contact line became pinned due to the shape changes of the solid-air interface ${ }^{35}$ referred to as the canthotaxis effect. ${ }^{36}$ Further elevated hydrostatic pressures resulted in sagging of the water-air interface into the groove between the secondary granules, due to the sustained pinning of the three-phase contact line at the edges. When the expanding water-air interface approached the next adjacent primary granules, the fluid front jumped over without soaking the nanocavities in between the primary granules. The liquid front discontinuously penetrated the microscopic groove between the secondary granules in discrete slip-stick steps that resulted from pinning and sag-transition phenomena. The observed dynamics was similar for hydrophilic and hydrophobic surfaces. In both cases, nanoplastrons remained stuck inside the nanocavities. Furthermore, in line with experimental data, we found that the described disappearance of the plastron inside the microscopic grooves was reversible (for further details, see Supplementary Section 4 and Supplementary Figure S3). The robustness of the remaining nanoplastrons formed the last barrier against complete wetting of the solid surface, that is, the Wenzel state. The dynamics of the final transition step is depicted in the panel on the right hand side in Figure 3c. The nanoplastron inside the hydrophilic cavity is less stable and collapsed at lower hydrostatic pressures than the remained plastron inside the hydrophobic cavity in accordance with the experimental results. In sum, the evolved nanotopography of springtail skin caused wetting to occur as a two-stage Cassie-Wenzel transition process, where the advancing liquid front penetrated the microscopic grooves between adjacent secondary granules before the remaining plastrons inside the nanocavities collapsed. In the absence of overhangs, the Cassie-Wenzel transition was found to occur in one step without remaining nanoplastrons (see lower panel in Figure 3c) and occurred-as observed experimentally-immediately for hydrophilic surfaces and at an elevated pressure for hydrophobic surfaces.

\section{DISCUSSION}

Our combined approach of experiment and simulation (Figures 1-3) provides new insights into the structural and chemical origins of the protection of springtail skin against wetting: Enforced wetting of a series of nanoimprinted polymer replicas and numerical FEM simulations clearly identified the nanoscopic primary granules of the cuticle to have a decisive role in liquid repellence. Specifically, the overhangs of the nanoscopic granules were concluded to effectively retain air (nanoplastrons) upon wetting, resulting in a solely structural wetting barrier even for liquids with low surface tension. Thus, our data prove the recently proposed geometrical control over wetting of Collembola skin, ${ }^{10}$ supporting and extending findings on engineered omniphobic surfaces. ${ }^{37-39}$ Furthermore, the CassieWenzel transition at elevated pressures was observed to occur as a stepwise process, enabling a reversible partial wetting due to nanoplastrons remaining entrapped inside the skin nanocavities, which facilitates recovery of the de-wetted state upon pressure reduction. The resulting last barrier against complete wetting can, for example, effectively protect springtails against suffocation in rain (rain droplets have a dynamic pressure in the range from 100 to $1000 \mathrm{hPa}) .{ }^{40}$ As wetting is a prerequisite for the attachment of microorganisms, the minimized wetting area similarly restricts the settlement of various bacteria and fungi occurring in the habitat of springtails.

Taken together, our studies revealed that the protective design principle of springtail skin critically depends on the nanotopography of the cuticle. We show that even intrinsically hydrophilic materials can resist wetting when structured according to this principle. Combining the nanoarchitecture of springtail skin with hydrophobic coatings was demonstrated to increase the barrier against wetting still 
further-beyond the characteristics of the natural template. The newly garnered understanding of the protective topography could pave the way for synthetic materials that effectively and durably resist wetting and biofouling.

\section{CONFLICT OF INTEREST}

The authors declare no conflict of interest.

\section{ACKNOWLEDGEMENTS}

We are grateful to Julia Nickerl for substantial support concerning the biology of Collembola, the TEM investigations and collecting the animals. We also thank Jannik Bäumer for collecting animals and assistance in preparing the polymer replicas. Furthermore, we thank Roland Vogel for measuring the shear viscosity of the used prepolymers. Hans-Georg Braun and Karina Grundke are gratefully acknowledged for helpful discussions throughout the work.

1 Hopkin, S. P. Biology of the Springtails (Insecta: Collembola) (Oxford University Press, USA, 1997).

2 Rusek, J. Biodiversity of collembola and their functional role in the ecosystem. Biodivers. Conserv. 7, 1207-1219 (1998).

3 Bellinger, P., Christiansen, K. \& Janssens, F. Collembola species catalogue. Checklist of the Collembola of the World http://www.collembola.org. Accessed 12 June 2012 (1998-2012).

4 Davies, W. M. On the tracheal system of collembola, with special reference, to that of sminthurus viridis, lubb. Q. J. Microsc. Sci. 71, 15-30 (1927).

5 Zinkler, D. Vergleichende untersuchungen zur atmungs-physiologie von collembolen (apterygota) und andereen bodenkleinarthropoden. Z. Vergl. Physiol. 52, 99-144 (1966).

6 Noble-Nesbitt, J. Transpiration in podura aquatica I. (collembola, isotomidae) and wetting properties of its cuticle. J. Exp. Biol. 40, 681-700 (1963).

7 Joosse, E. N. G. \& Groen, J. B. Relationship between saturation deficit and survival and locomotory activity of surface dwelling collembola. Entomol. Exp. Appl. 13, 229-235 (1970).

8 Ghiradella, H. \& Radigan, W. Collembolan cuticle - wax layer and anti-wetting properties. J. Insect. Physiol. 20, 301-306 (1974).

9 King, P. E., Pugh, P. J. A., Fordy, M. R., Love, N. \& Wheeler, S. A. A comparison of some environmental adaptations of the littoral collembolans anuridella marina (willem) and anurida maritima (guérin). J. Nat. Hist. 24, 673-688 (1990).

10 Helbig, R., Nickerl, J., Neinhuis, C. \& Werner, C. Smart skin patterns protect springtails. PLOS ONE 6, e25105 (2011).

11 Hale, W. G. \& Smith, A. L. Scanning electron microscope studies of cuticular structures in the genus onychiurus (collembola). Rev. Ecol. Biol. Sol. 52, 343-354 (1966).

12 Cassie, A. B. D. \& Baxter, S. Wettability of porous surfaces. Trans. Faraday Soc. 40, 546-551 (1944).

13 Nakajima, A. Design of hydrophobic surfaces for liquid droplet control. NPG Asia Mater. 3, 49-56 (2011).

14 Wenzel, R. Resistance of solid surfaces to wetting by water. Ind. Eng. Chem. Res. 28, 988-994 (1936).

15 Lafuma, A. \& Quéré, D. Superhydrophobic states. Nat. Mater. 2, 457-460 (2003).

16 Quéré, D. Wetting and roughness. Annu. Rev. Mater. Res. 38, 71-99 (2008).

17 Barbieri, L., Wagner, E. \& Hoffmann, P. Water wetting transition parameters of perfluorinated substrates with periodically distributed flat-top microscale obstacles. Langmuir 23, 1723-1734 (2007).

18 Nosonovsky, M. Multiscale roughness and stability of superhydrophobic biomimetic interfaces. Langmuir 23, 3157-3161 (2007).

19 Bormashenko, E. Wetting transitions on biomimetic surfaces. Phil. Trans. A Math. Phys. Eng. Sci. 368, 4695-4711 (2010).
20 Verho, T., Korhonen, J. T., Sainiemi, L., Jokinen, V., Bower, C., Franze, K., Franssila, S. Andrew, P., Ikkala, O. \& Ras, R. H. A. Reversible switching between superhydrophobic states on a hierarchically structured surface. Proc. Natl Acad. Sci. USA 109, 10210 10213 (2012).

21 Neinhuis, C. \& Barthlott, W. Characterization and distribution of water-repellent, selfcleaning plant surfaces. Ann. Bot. 79, 667-677 (1997).

22 Gao, X. \& Jiang, L. Biophysics: water-repellent legs of water striders. Nature 432, 36 (2004).

23 Bush, J. W. M., Hu, D. L. \& Prakash, M. The integument of water-walking arthropods: Form and function. Adv. Insect Physiol. 34, 117-192 (2007).

24 Koch, K., Bhushan, B. \& Barthlott, W. Diversity of structure, morphology and wetting of plant surfaces. Soft Matter 4, 1943-1963 (2008).

25 Chou, S. Y., Krauss, P. R. \& Renstrom, P. J. Imprint lithography with 25-nanometer resolution. Science 272, 85-87 (1996).

26 Williams, S. S., Retterer, S., Lopez, R., Ruiz, R., Samulski, E. T. \& DeSimone, J. M High-resolution pfpe-based molding techniques for nanofabrication of high-pattern density, sub-20 nm features: A fundamental materials approach. Nano Lett. 10, 1421-1428 (2010)

27 LaFratta, C. N., Baldacchini, T., Farrer, R. A., Fourkas, J. T., Teich, M. C., Saleh, B. E. A. \& Naughton, M. J. Replication of two-photon-polymerized structures with extremely high aspect ratios and large overhangs. J. Phys. Chem. B 108, 11256-11258 (2004)

28 LaFratta, C. N., Li, L. \& Fourkas, J. T. Soft-lithographic replication of 3d microstructures with closed loops. Proc. Natl Acad. Sci. USA 103, 8589-8594 (2006).

29 Schulte, A. J., Koch, K., Spaeth, M. \& Barthlott, W. Biomimetic replicas: Transfer of complex architectures with different optical properties from plant surfaces onto technical materials. Acta Biomater. 5, 1848-1854 (2009).

30 Pulsifer, D. P., Lakhtakia, A., Martin-Palma, R. J. \& Pantano, C. G. Mass fabrication technique for polymeric replicas of arrays of insect corneas. Bioinspir. Biomim. $\mathbf{5}$ 036001 (2010)

31 Ko, D.-H., Tumbleston, J. R., Henderson, K. J., Euliss, L. E., DeSimone, J. M., Lopez, R. \& Samulski, E. T. Biomimetic microlens array with antireflective "moth-eye" surface. Soft Matter 7, 6404-6407 (2011).

32 Zhang, T., Li, M., Su, B., Ye, C., Li, K., Shen, W., Chen, L., Xue, Z., Wang, S. \& Jiang L. Bio-inspired anisotropic micro/nano-surface from a natural stamp: grasshopper wings. Soft Matter 7, 7973-7975 (2011).

33 Tavana, H., Petong, N., Hennig, A., Grundke, K. \& Neumann, A. Contact angles and coating film thickness. J. Adhes. 81, 29-39 (2005).

34 Aland, S., Lowengrub, J. \& Voigt, A. Two-phase flow in complex geometries: a diffuse domain approach. Comput. Model. Eng. Sci. 57, 77-107 (2010).

35 Oliver, J. F., Huh, C. \& Mason, S. G. Resistance to spreading of liquids by sharp edges. J. Colloid Interface Sci. 59, 568-581 (1977).

36 Berthier, J., Loe-Mie, F., Tran, V., Schoumacker, S., Mittler, F., Marchand, G. \& Sarrut, N. On the pinning of interfaces on micropillar edges. J. Colloid Interface Sci. 338 , 296-303 (2009).

37 Tuteja, A., Choi, W., Ma, M. L., Mabry, J. M., Mazzella, S. A., Rutledge, G. C., McKinley, G. H. \& Cohen, R. E. Designing superoleophobic surfaces. Science $\mathbf{3 1 8}$ 1618-1622 (2007).

38 Cao, L. L., Hu, H. H. \& Gao, D. Design and fabrication of micro-textures for inducing a superhydrophobic behavior on hydrophilic materials. Langmuir 23, 4310-4314 (2007)

39 Tuteja, A., Choi, W., Mabry, J. M., McKinley, G. H. \& Cohen, R. E. Robust omniphobic surfaces. Proc. Natl Acad. Sci. USA 105, 18200-18205 (2008).

40 Zheng, Q. S., Yu, Y. \& Zhao, Z. H. Effects of hydraulic pressure on the stability and transition of wetting modes of superhydrophobic surfaces. Langmuir 21 12207-12212 (2005)

(c) (1) $\odot$ This work is licensed under a Creative Commons Attribution-NonCommercial-NoDerivs 3.0 Unported view a copy of this license, visit http://creative License. To view a copy of this
commons.org/licenses/by-nc-nd/3.0/

Supplementary Information accompanies the paper on the NPG Asia Materials website (http://www.nature.com/am) 